\title{
Modeling the composition of fine-grained modified concrete elaborated with vibropressing technology
}

\author{
E. R. Pyataev ${ }^{1, *}$, and A. Y.Ushakov ${ }^{1}$ \\ ${ }^{1}$ Moscow State University of Civil Engineering, 129337, Yaroslavskoye shosse, 26, Moscow, Russia
}

\begin{abstract}
The article analyzes the property requirements of small-piece products used in road construction, specially those related to water and frost resistance. It describes the main features of the vibration technologies involved in the manufacture of building materials, in particular, paving slabs. The article presents conclusive results, obtained through scientific research, which allow us to establish the influence of both, the costs of the main components and the parameters of vibration compression, on the properties of the final product. Combining the analysis of classical methods with the results of experimental research, a methodology has been developed for reaching an optimal composition of fine-grained vibropressed concrete modified with active additives. It is shown that the particular strength achieved from the combination of vibroformed fine-grained concrete, whose average density is $2270-2320 \mathrm{~kg} / \mathrm{m} 3$, with polymer multifunctional modifiers, is mainly due to the use of Portland cement, a modifying additive, and the pressure conditions employed during the experiment..
\end{abstract}

\section{Introduction}

During installation and throughout their lifespan, road products are subjected to intense mechanical stresses, freezing and thawing, alternated with moistening and drying, as well as the effects of salt solutions, including sodium chloride, gasoline and technical oils, etc. That is the reason for the high compressive strength (class not less than B30), frost resistance (grade for frost resistance not lower than F200) and water absorption (not more than 6\% by weight) [1-3] demanded from these products. It should be noted that due to the current conditions of increasing traffic and pollution of the urban environment, a proper performance requieres that the characteristics of these products, used in parking lots of motor vehicles and gas stations, exced the normative [4-6].

In this context, we will focus on the use of paving slabs, whose main method of manufacture is vibrating technology [7-9], and which have been in steady demand across a large number of russian cities, as their use has been encouraged by large-scale national programs for the improvement of urban layouts and communication routes.

Vibration technology, or more precisely, molding under pressure plus simultaneous mechanical action, has been hystorically implemented in the manufacture of hollow concrete

\footnotetext{
${ }^{*}$ Corresponding author: pyatay92@mail.ru
} 
wall blocks, curbs, cement tiles and wall or pavement blocks. The main component of all these final products is concrete: expanded-clay concrete gravel for concrete blocks, finegrained concrete for pavement blocks and road kerbs, or fiber concrete for wall panels [7-9]. In addition, another common characteristic present in this group of products is the use of special additives (plasticizing, water-repellent), which provide optimal conditions for vibroforming and the attainment of high-quality products [10-12].

Because of it, one of the most important aspects of vibropressing technology is the study of the relationship between prescription factors and factors that regulate the molding mode $[13,14]$.

\section{Experimental}

The goal of our research was the formalization of an experiment aimed towards setting the foundations of the technology and developing methods necessary to select the optimal composition of paving blocks, based on vibroformed fine-grained concrete with multifunctional modifiers, for the conditions previously described. The experimental conditions are given in table 1.

Table 1. The conditions of the experiment

\begin{tabular}{|l|c|c|c|c|c|}
\hline \multicolumn{1}{|c|}{ Indicator } & \multirow{2}{*}{$\begin{array}{c}\text { Symbol } \\
\mathbf{X}_{\mathbf{i}}\end{array}$} & $\begin{array}{c}\text { Average } \\
\text { value, } \\
\mathbf{X}_{\mathbf{i}}\end{array}$ & $\begin{array}{c}\text { The range } \\
\text { of variation, } \\
\mathbf{\Delta X}_{\mathbf{i}}\end{array}$ & \multicolumn{2}{|c|}{$\begin{array}{c}\text { The value of } \\
\text { the indicator at } \\
\text { levels }\end{array}$} \\
\cline { 5 - 6 } & & 360 & 40 & 300 & 380 \\
\hline $\begin{array}{l}\text { The consumption of Portland } \\
\text { cement, } \mathrm{kg} / \mathrm{m}^{3}\end{array}$ & $\mathrm{X}_{1}$ & 1,0 & 0,2 & 0,8 & 1,2 \\
\hline Plasticizer consumption,\% & $\mathrm{X}_{2}$ & 5,0 & 0,4 & 4,6 & 5,4 \\
\hline $\begin{array}{l}\text { Water- repellent agent } \\
\text { consumption,\% }\end{array}$ & $\mathrm{X}_{3}$ & & & & \\
\hline Pressing pressure, MPa & $\mathrm{X}_{4}$ & 0,15 & 0,05 & 0,10 & 0,20 \\
\hline
\end{tabular}

\section{Results section}

The mathematical processing of the experimental results, made it possible to obtain, through regression, general equations for the compressive strength (Y1) and the average density (Y2) which can be expected from further applicated research. The following mathematical models (polynomials) were obtained:

- for compressive strength:

$$
\mathrm{Y} 1=37,2+2,8 \mathrm{X} 1+2,0 \mathrm{X} 2+1,7 \mathrm{X} 3+1,6 \mathrm{X} 4+1,4 \mathrm{X} 1 \mathrm{X} 3-0,9 \mathrm{X} 2 \mathrm{X} 4-1,2 \mathrm{X} 42
$$

- for medium density:

$$
\mathrm{Y} 2=2415+21 \mathrm{X} 1-14 \mathrm{X} 2+12 \mathrm{X} 3+10 \mathrm{X} 1 \mathrm{X} 3+10 \mathrm{X} 22
$$

Regarding the confidence intervals chosen to determine the degree of significance of the coefficients linked to our variables, the value for $\mathrm{Y} 1$ has been determined to be $\Delta \mathrm{b} 1=0.8$, and $\Delta \mathrm{b} 2=8.4$ for $\mathrm{Y} 2$. All coefficients out of the confidence intervals are assumed to be insignificant and equal to zero. 
An explanatory analysis of the meaning of the polynomial equations allows us to formulate the following conclusions:

- Pressure applied during the creation of concrete derivates has the greatest effect on strength and on their average density.

- The effects of the use of modifying additives and the pressure exerted are inversely proportional, meaning that the maximum strength of the product will be achieved through the equilibrium between pressure and the optimal consumption of polycarboxylate additives.

- A synergistic, multiplicative effect can be observed when a water-repellent agent is combined with an strong flow of plasticizer.

\section{Discussion section}

We can conclude that the biggest part of the strength attained by the samples, of vibroformed fine-grained concrete with polymer multifunctional modifiers, is due to the use of Portland cement (coefficient at X1 equal to 2.8), and therefore an increase in its consumption leads to an increase in compressive strength.

The influence of the presence of a water-repellent agent (X3) on the strength and density of the product is manifested to a lesser degree, for the change intervals indicated in the table 1. However, when it appears combinated with Portland cement, these components show a synergistic effect that manifests itself in pairwise interaction (coefficient at X1X3 equal to 1.4): the increase in strength occurs more intensively than in comparison with the individual effect of each factor on the result. In our opinion, this is due to the conditions of physicochemical and chemical interactions at the grain boundaries of the fine aggregate, occurred during the simultaneous vibration and stamping pressure requiered by production.

Finally, the influence of pressure is manifested both as an independent factor (coefficients in X4 and X42), and as a factor of joint influence (coefficient in X2X4).

A deeper interpretation reveals that sign of the coefficient of X2X4 (- 0.9) indicates the presence of an inversely proportional relationship between the overspending of plasticizer, which provides excessive plasticization in the preparation of the mixture, and the pressure employed. With an increase in the values of both factors, the strength increases inversely (is actually reduced). Similarly, an increase in the consumption of the modifying additive (within the limits allowed by the experimental conditions), allows the pressure to be reduced.

The effect of the use of a plasticizer, on average concrete density, and pressure on strength, is extreme (the coefficients at X22 and X42 are 8 and -0.9, respectively), which makes it advisable to use the Method of analytical optimization [14-16], developed at MGSU, and repeatedly used during the processing of the experimental results when solving technological problems of various types, and while developing methods for choosing the composition of multicomponent raw materials.

This method applies on the previously obtained regressive polynomial equations, result of processing the experimental data, to obtain, using methods of mathematical analysis, local optima. Processing and optimization of the results was carried out with a software package for computers.

To find the local extremes of the function $\mathrm{Y} 2=(\mathrm{X} 1, \mathrm{X} 2, \mathrm{X} 3)$ we calculate its partial derivative with respect to $\mathrm{X} 2$ and equate the result to 0 , to find the value of $\mathrm{X} 2$ corresponding to the minimum density:

$$
\partial Y_{2} / \partial X_{2}=-14+20 X_{2}=0 \rightarrow X_{2}=\frac{14}{20}=0,7
$$

We find the local extremes of the function $\mathrm{Y} 1=(\mathrm{X} 1, \mathrm{X} 2, \mathrm{X} 3, \mathrm{X} 4)$ in the same way: we differentiate the function with respect to $\mathrm{X} 4$ and equal the derivative to zero: 


$$
\partial Y_{1} / \partial X_{4}=1,6+1,0 X_{2}-2,4 X_{4}=0 \rightarrow X_{4}=0,66+0,4 X_{2}=0,66+0,4 \times 0,7=0,94
$$

Solving the equations $\mathrm{Y} 1(\mathrm{X} 1, \mathrm{X} 2, \mathrm{X} 3, \mathrm{X} 4)$ and $\mathrm{Y} 2(\mathrm{X} 1, \mathrm{X} 2, \mathrm{X} 3)$ at $\mathrm{X} 4=0.94+0.5 \mathrm{X} 2$ where $\mathrm{X} 2=0.70$, we obtain the following optimization functions:

- For compressive strength:

$$
\mathrm{Y}_{1}=40,8+2,8 \mathrm{X}_{1}+1,7 \mathrm{X}_{3}+1,4 \mathrm{X}_{1} \mathrm{X}_{3}
$$

- For medium density:

$$
\mathrm{Y}_{2}=2409+21 \mathrm{X}_{1}+12 \mathrm{X}_{3}+10 \mathrm{X}_{1} \mathrm{X}_{3}
$$

For an easier interpretation, we carried out a graphical representation of the dependence between the compressive strength on the flow rate of Portland cement (X1) and flow rate of water-repellent agent (X3). As a result, we obtain a nomogram (Fig. 1) to help us predict the strength of fine-grained vibroformed concrete or choose the optimal component consumption.

A graphical interpretation of the dependence of concrete strength (MPa) on the flow rate of the fine-grained fraction, the flow rate of the water repellent and the optimized pressing pressure of $0.2 \pm 0.05 \mathrm{MPa}$ is presented in Fig. 1

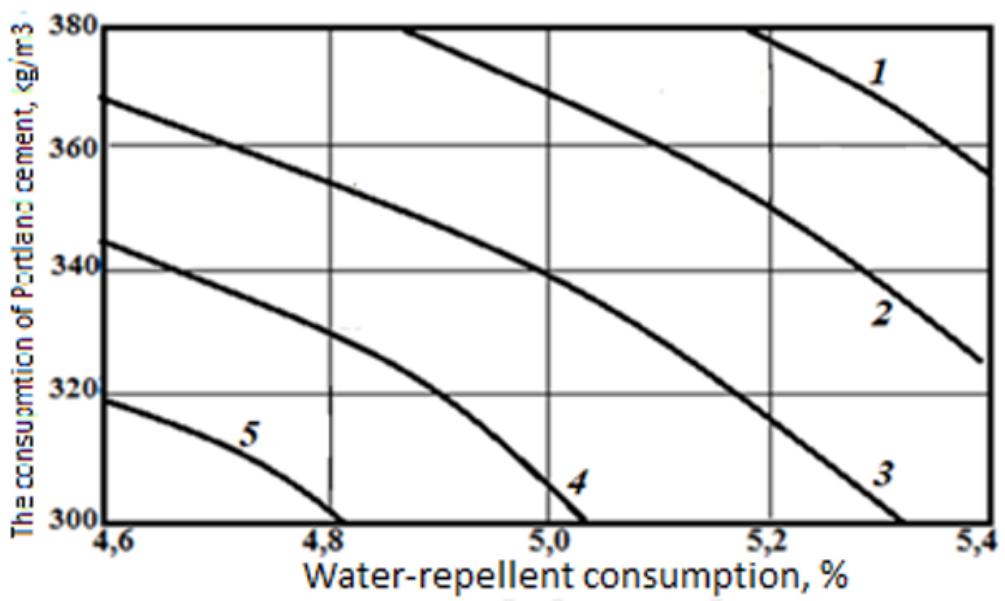

Fig. 1. The dependence of the strength of concrete $(\mathrm{MPa})$ on the flow rate of the fine-grained fraction and the flow rate of the water repellent agent at a pressing pressure of $0.2 \pm 0.05 \mathrm{MPa}: 1-44 ; 2$ to $42 ; 3$ to $40 ; 4$ to $36 ; 5-34$

The main tasks accomplished during the development of a new technology of concrete products or the modernization of existing ones should be the development of a method for selecting the composition of base concrete and the optimization of technological parameters.

The nomogram obtained, as a result of processing the data obtained during an active experiment, confirmed our initial hypothesis that the strength of vibroformed fine-grained concrete with polymer multifunctional modifiers (density of 2270-2320 kg/m3) depends mainly on the consumption of Portland cement and a modified additive as well as on the pressing conditions, allowing us to evaluate the dependence of the strength on these factors to choose their optimal ratio. 
At a loading pressure of less than $0,1 \mathrm{MPa}$, it is not enough to seal the system, and at a pressure of more than 0,2 MPa, the maximum density is also not achieved (Fig. 2).

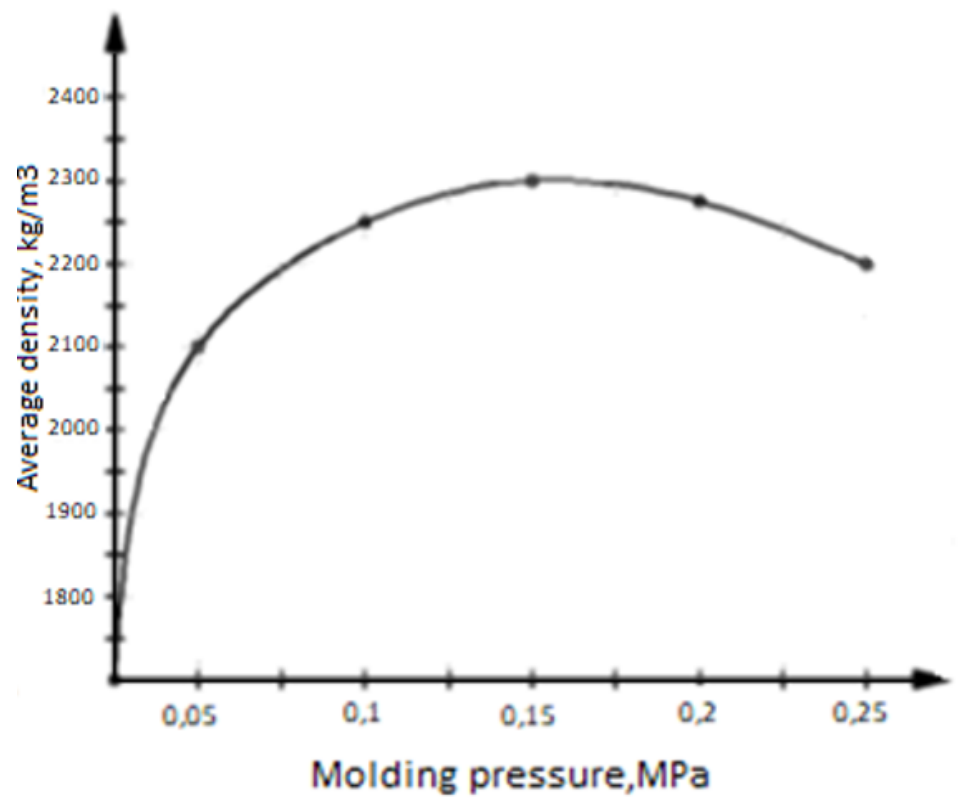

Fig. 2 The average density of freshly formed paving slab samples rom the pressure value

\section{Conclusion}

It has been established that, in the intervals of changes in factors under consideration, both the composition characteristics and the vibration forming parameters have had the greatest influence on the result. It is very important to obtain the highest achievable strength of the slabs for paving, by taking into account the combined effect of the flow of modifying additives and pressure. With that in mind, the final conclusion is that the optimal consumption of polycarboxylate additives, in the context of our experiment, should be $1,0 \pm 0,2 \%$ and the pressure 0,2 $\pm 0,05 \mathrm{MPa}$.

The results of the experiment which are presented in the article allows us to form the basis of the methodology for selecting the composition of paving blocks based on fine-grained concrete modified by polymers. The selection and optimization of vibration forming parameters could be carried out in additional studies.

Work carried out on the frame of the plan for research works "NRU MSUCE" and according to the basis of the initiative.

\section{References}

1. A.D. Zhukov, N.V. Naumova, A.S. Chkunin, Shrinkage in the formation of cellular concrete structure. Internet herald VolgGASU. № 4 (2014)

2. B.M. Rumyantsev, A.D. Zhukov, A.V. Chugunkov, D.I. Aristov, Optimization of cellular structures. Scientific Review. № 13. P. 128-131 (2015) 
3. S. A. Tkach, V. I. Telichenko, Solution of ecological problems during the disposal of industrial waste in the production of aerated concrete. Ecology of urbanized territories in Moscow. № 2. P. 39-44. (2016)

4. Gusev B.V., Gubanov D.A., Gubanova O.Yu. Erofeev O.Yu. Creation of building composite materials on the basis of substandard concrete for various technologies // Proceedings of the III All-Russian (II International) conference "Concrete and reinforced concrete - a look into the future". M., 2014, MSSU, vol. VI. from. 265-273

5. Gusev B.V., Kudryavtseva V.D., Tarnosyn K.V. Secondary use of concrete// International scientific and technical conference "Development of effective aviation, industrial, electrotechnical and building materials and research of their durability in conditions of influence of various operational factors". Saransk, Mordovian University, 2013. pp. 21-25

6. Gusev B., Falikman V. Structural concrete in the age of sustainable development / An ACI Technical Publication. Durability and Sustainability of Concrete Structures Workshop Proceedings Italy, ACI, 2015. pp. 36.1-36.8

7. B.M. Rumiantcev, A.D. Zhukov, E.Yu. Bobrova, I. P. Romanova, D.B. Zelenshikov, T.V. Smirnova, The systems of insulation and a methodology for assessing the durability. MATEC Web of Conferences. Vol. 86 (2016)

8. A.D. Zhukov, A.V. Chugunkov, A.O. Khimich, Cellular concrete for monolithic constructions. Industrial and civil construction. № 3. P. 21-23 (2013)

9. A.D. Zhukov, N.V. Naumova, R.M. Mustafayev, N.A. Mayorova, Modeling of properties of highly porous materials of a combined structure. Industrial and civil construction. № 7. P. 48-51 (2014)

10. I.J. Gnip, V.J. Keršulis, S.J. Vaitkus, Predicting the deformability of expanded polystyrene in long-term compression. Mechanics of Composite materials; 41(5):407414 (2005)

11. B.M. Rumyantsev, A.D. Zhukov, T.V. Smirnova, Energy efficiency and methodology for creating heat insulation materials. Internet-Bulletin of VolgGASU. №. 4 (35). C. 3 (2014)

12. A.D. Zhukov, M.O. Asamatdinov, A.S. Chkunin, K.K. Ivanov, G.B. Rumyantsev, Wall materials based on local raw materials. Innovations in life. № 4 (19). P. 35-43 (2016)

13. A.D. Zhukov, A.S. Chkunin, A.O. Khimich, D.I. Aristov, M.S. Novikova, Stress state in the technology of honeycomb materials. Scientific review. № 7. P. 218-221 (2015)

14. A.D. Zhukov, A.V. Chugunkov, Local analytical optimization of technological processes. Herald MSUCE. № 1-2. P. 273-278 (2011)

15. B.M. Rumyantsev, A.D. Zhukov, A.V. Chugunkov, D.I. Aristov, Optimization of cellular structures. Scientific Review. № 13. P. 128-131 (2015)

16. B.M. Rumiantcev, A.D. Zhukov, D.B. Zelenshikov, A.S. Chkunin, K.K. Ivanov, Yu.V. Sazonova. Insulation systems of the building construtions. MATEC Web of Conferences. Vol. 86 (2016) 